\title{
Fermented Cordyceps cicadae Mycelia Extracts Ameliorate Dry Eye Symptoms through Reduction of Cornea Epithelial Cell Apoptosis and Maintenance of Conjunctival Goblet Cells in a Mouse Dry Eye Model
}

\author{
Tung-Yu Lin ${ }^{1, \#}$, Han-Hsin Chang ${ }^{2, \#}$, Yu-Jun Tang ${ }^{3}$, Chin-Chu Chen ${ }^{4,5}$, Li-Ya Lee ${ }^{6}$, David Pei-Cheng Lin ${ }^{1,7, *}$ \\ ${ }^{1}$ Department of Medical Laboratory and Biotechnology, Chung Shan Medical University, Taichung City, Taiwan \\ ${ }^{2}$ Department of Nutrition, Chung Shan Medical University, Taichung City, Taiwan \\ ${ }^{3}$ Institute of Biochemistry, Microbiology, and Immunology, Chung Shan Medical University, Taichung City, Taiwan \\ ${ }^{4}$ Institute of Food Science and Technology, National Taiwan University, Taiwan \\ ${ }^{5}$ Department of Food Science, Nutrition, and Nutraceutical Biotechnology, Shih Chien University, Taiwan \\ ${ }^{6}$ Department of Veterinary Medicine, National Chung Hsin University, Taiwan \\ ${ }^{7}$ Department of Ophthalmology, Chung Shan Medical University Hospital, Taichung City, Taiwan \\ \#These are first authors. \\ *Corresponding author: pcl@csmu.edu.tw
}

\begin{abstract}
Cordyceps cicadae $(\mathrm{Cc})$, a traditional Chinese medicine, has been shown to possess immunomodulatory and anti-inflammatory activities, and is regarded as having effects in vision improvement, but with no reported evidence. This study investigated the effects of Cordyceps cicadae fermented mycelia extracts (Cc extracts) in a benzalkonium chloride (BAC)-induced mouse dry eye model. Female ICR mice aged 6 weeks were randomly divided into four groups: blank, BAC-damaged without Cc extracts, BAC-damaged with $10 \mathrm{mg} / \mathrm{kg}$ bodyweight of $\mathrm{Cc}$ extracts, BAC-damaged with $100 \mathrm{mg} / \mathrm{kg}$ bodyweight of Cc extracts. The results showed that tear volume, tear film breakup time, and cornea surface indexes, including smoothness, opacity, topography, and the extent of lissamine green staining, were all improved with intake of Cc extracts intake, when compared to the status of the BAC-damaged group without $\mathrm{Cc}$ extracts. Immunohistochemical assays showed moderate change of $\mathrm{Ki}-67^{+}$and $\mathrm{Np} 63^{+}$epithelial cell populations, while apoptotic epithelial cells, as detected by TUNEL assay, were decreased. PAS stain showed that the conjunctival goblet cell number and total cell area were decreased in the BAC-damaged group with $\mathrm{Cc}$ extracts at $10 \mathrm{mg} / \mathrm{kg}$ bodyweight. This study demonstrated that $\mathrm{Cc}$ extracts effectively ameliorate BAC-induced dry eye symptoms through enhancement of cornea resilience against BAC-induced damages and maintenance of conjunctival goblet cells.
\end{abstract}

Keywords: Cordyceps cicadae, mycelia extracts, amelioration, dry eye symptoms, cornea epithelial cell apoptosis, conjunctival goblet cells

Cite This Article: Tung-Yu Lin, Han-Hsin Chang, Yu-Jun Tang, Chin-Chu Chen, Li-Ya Lee, and David Pei-Cheng Lin, "Fermented Cordyceps cicadae Mycelia Extracts Ameliorate Dry Eye Symptoms through Reduction of Cornea Epithelial Cell Apoptosis and Maintenance of Conjunctival Goblet Cells in a Mouse Dry Eye Model." Journal of Food and Nutrition Research, vol. 5, no. 5 (2017): 320-330. doi: 10.12691/jfnr-5-5-6.

\section{Introduction}

Dry eye disease (DED) prevails between $7.4 \%$ and $33.7 \%$ among human populations [1] and it was estimated that $25 \%$ of patients who visited ophthalmic clinics due to symptoms of dry eye [2]. The risk factors of DED include age, gender, autoimmune disease, long-term contact lens wearing, refractive laser surgery, excessive visual task performance, intake of certain systemic medications, smoking, and low humidity environments [2,3]. These risk factors characterize DED as a chronic ocular surface disorder that affects significantly quality of life through enduring discomfort and visual disturbances. Due to such extended impact, DED has attracted much attention and many efforts have been exerted in finding the ways to alleviate its symptoms.

DED is a multifactorial disease that is manifested in many aspects. Previous studies indicated that inflammation, such as seen in systemic autoimmune diseases including Sjogren's syndrome and systemic lupus erythematosus, is a common manifestation of dry eye symptoms [4,5]. Some researchers have thus proposed to use topical or systemic immunomodulatory therapy in severe dry eye conditions $[6,7]$. Increase of pro-inflammatory markers with excessive oxidants in the tear is also a hallmark of DED $[8,9]$. Thus, anti-inflammation by use of anti-oxidants, 
such as the use of dietary alpha-lipoic acid or topic administration of CoQ10, has been experimentally applied for dry eye symptom alleviation $[10,11,12]$.

Cordyceps cicadae $(\mathrm{Cc})$, a traditional Chinese health food, was shown to possess immunomodulatory activities [13] and anti-inflammatory effects [14]. These effects, if exerted in the eye, may help to relieve dry eye symptoms. Nevertheless, whether intake of $\mathrm{Cc}$ extracts may ameliorate dry eye symptoms is not known. This study investigated this potential effect in benzalkonium chloride (BAC)-induced mouse dry eye model [15].

\section{Results}

\subsection{Dietary Cc Extracts Ameliorate Ocular Surface Damages Induced by Benzalkonium Chloride}

The ocular surface damages were assessed by semiquantitative grading systems according to smoothness, topography, opacity, and the extent of corneal staining by lissamine green. The results showed that dietary intake of
Cc extracts helped to alleviate ocular surface damages in all of the four grading systems, except for opacity, while those without intake of $\mathrm{Cc}$ extracts showed significant damages on the ocular surface (Figure 1). The effective dose was $100 \mathrm{mg} / \mathrm{kg}$ of body weight and this effective dose was consistent for mitigation of the smoothness, topography, and corneal staining grading.

\subsection{Tear Production under the Influence of Cc Extracts}

Dietary intake of $\mathrm{Cc}$ extracts helped to increase the aqueous tear production after ocular surface damage by BAC (Figure 2). At day 7 after the BAC-induced damage, $\mathrm{Cc}$ extracts given at $100 \mathrm{mg} / \mathrm{Kg}$ of body weight lead to significant increase of tear production as compared to that of the BAC-damaged group. At day 10 after the damage, both groups given at $10 \mathrm{mg} / \mathrm{Kg}$ and $100 \mathrm{mg} / \mathrm{Kg}$ of body weight of $\mathrm{Cc}$ extracts showed significant increase of tear production. Notably, starting from day 4 , with intake of $\mathrm{Cc}$ extracts at $10 \mathrm{mg} / \mathrm{Kg}$ or $100 \mathrm{mg} / \mathrm{Kg}$, the tear production was higher than either the control or the BACdamaged group.
A
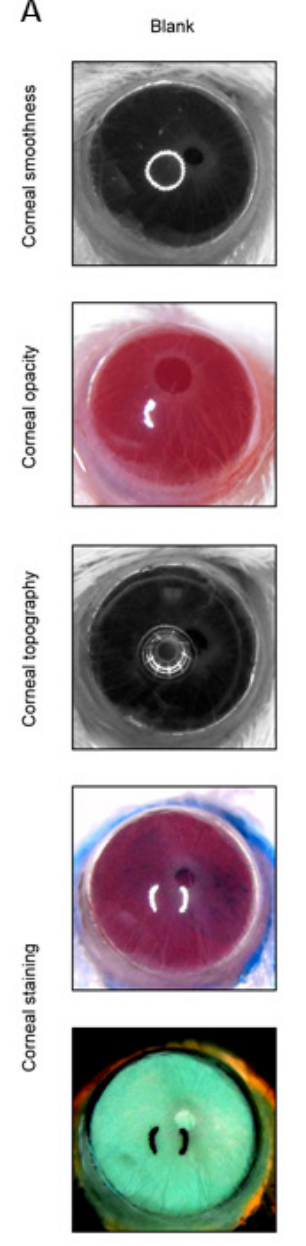
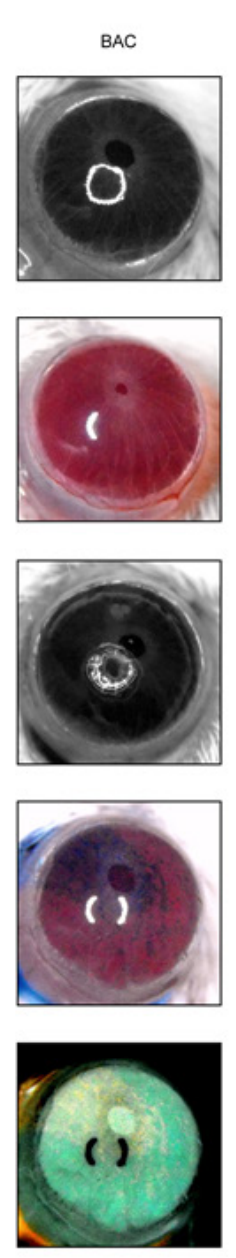
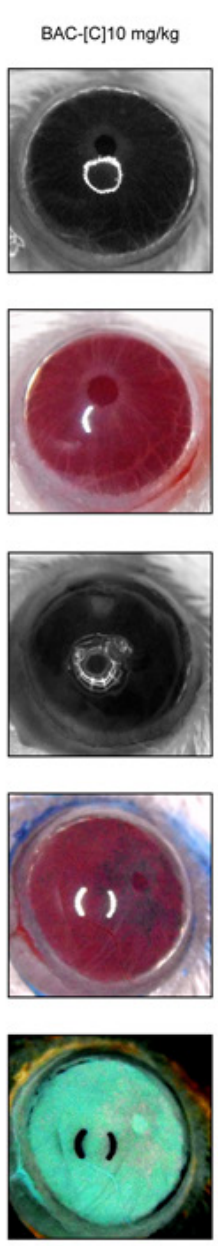

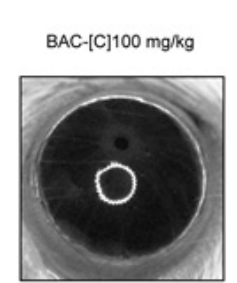

B

(1)
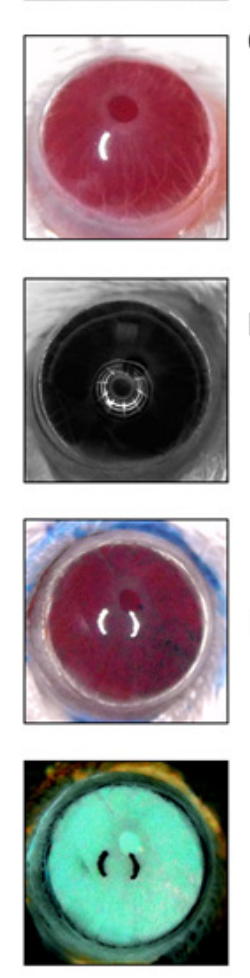
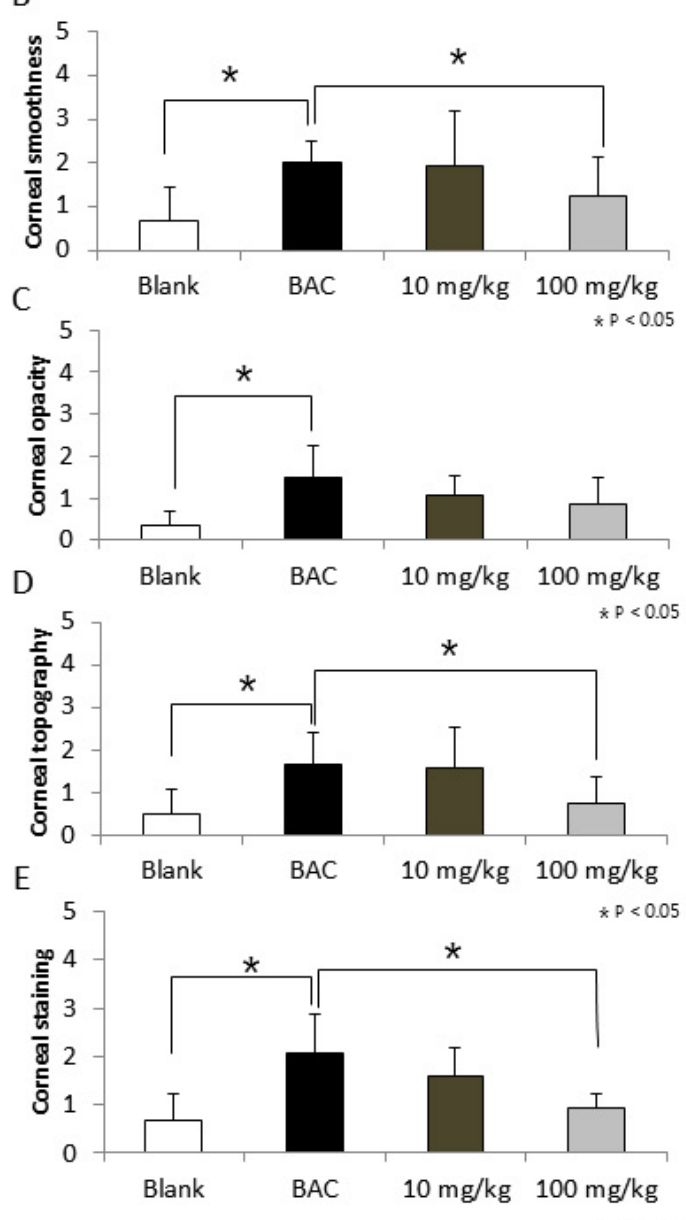

In A, less irregularity of reflective light was observed with intake of intake of Cc extracts. Negative images were also shown for the extent of lissamine green staining. In B, semi-quantitative analysis showed that the effective dose was at $100 \mathrm{~g} / \mathrm{Kg}$ of bodyweight. BAC: benzalkonium chloride-damaged group without intake of CC extracts. BAC-[C] $10 \mathrm{mg} / \mathrm{kg}$ and BAC-[C] $100 \mathrm{mg} / \mathrm{kg}$ : benzalkonium chloride-damaged groups with intake of CC extracts at $10 \mathrm{mg} / \mathrm{kg}$ and $100 \mathrm{mg} / \mathrm{Kg}$, respectively.

Figure 1. Ocular surface grading for corneal smoothness, opacity, topography, and extent of lissamine green staining at day 10 of the experiment 


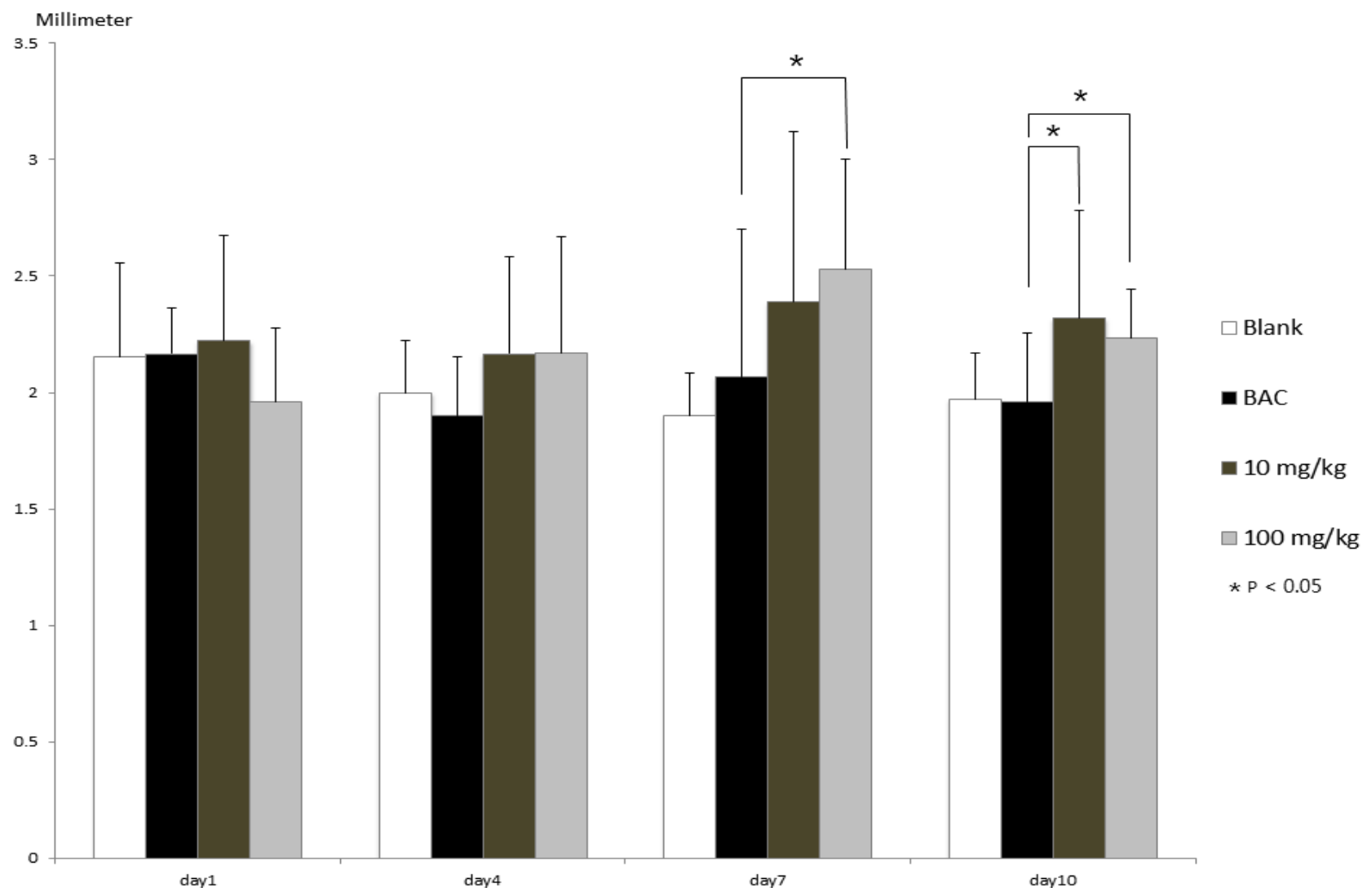

BAC: benzalkonium chloride-damaged group without intake of CC extracts. $10 \mathrm{mg} / \mathrm{kg}$ and $100 \mathrm{mg} / \mathrm{kg}$ : benzalkonium chloride-damaged groups with intake of Cordyceps cicadae mycelia fermented extracts at $10 \mathrm{mg} / \mathrm{kg}$ and $100 \mathrm{mg} / \mathrm{Kg}$, respectively.

Figure 2. Aqueous tear production (in millimeter) assessed with a 1-mm-width strip cut off from a $\mathrm{pH}$ test paper at days 1, 4, 7, and 10 of the experiment

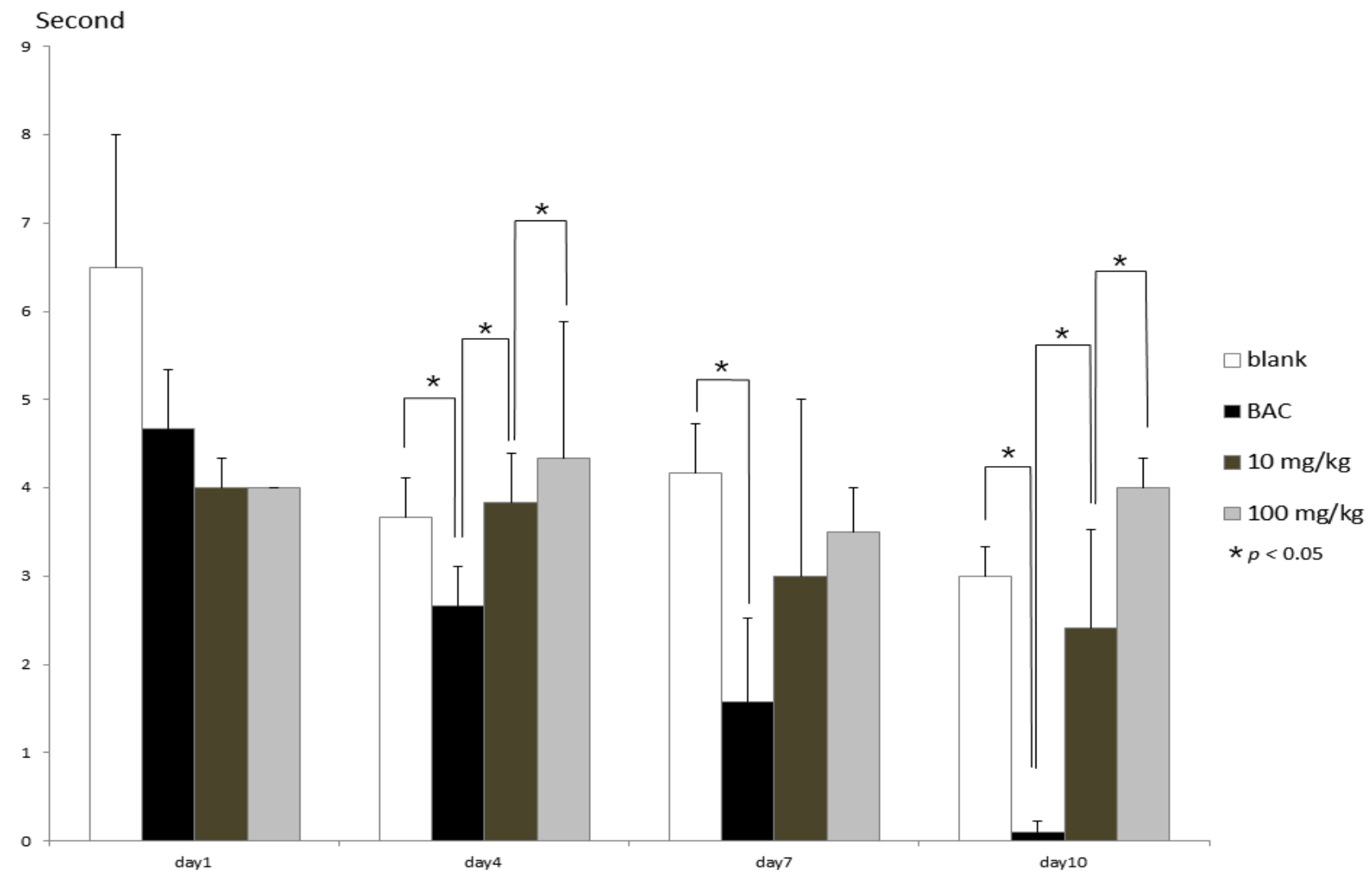

BAC: benzalkonium chloride-damaged group without intake of Cc extracts. $10 \mathrm{mg} / \mathrm{kg}$ and $100 \mathrm{mg} / \mathrm{kg}$ : benzalkonium chloride-damaged groups with intake of $\mathrm{CC}$ extracts at $10 \mathrm{mg} / \mathrm{kg}$ and $100 \mathrm{mg} / \mathrm{Kg}$, respectively.

Figure 3. Tear film breakup time (in seconds) assessed after fluorescein staining and recording at days 1, 4, 7, and 10 of the experiment 


\subsection{Increased Tear Film Breakup Time with Cc Extracts}

Since the tear volume was increased with intake of Cc extracts, we further assessed the tear quality as reflected by tear film breakup time (TBUT). The results showed that BAC caused significant reduction of TBUT at day 1 after the damage (Figure 3) and this detrimental effect aggravated more severely at days 4,7 , and further to day 10 . TBUT was significantly increased at day 4 with intake of Cc extracts in both $10 \mathrm{mg} / \mathrm{Kg}$ and $100 \mathrm{mg} / \mathrm{Kg}$ dose groups, as compared to that of the BAC-damaged group. However, the significance of increase did not persist to day 7 . At day 10, the TBUT increase regained its significance. Furthermore, the TBUTs of $100 \mathrm{mg} / \mathrm{Kg}$ dose group was significantly longer than that of the $10 \mathrm{mg} / \mathrm{Kg}$ dose group at both day 4 and day 10 .

\subsection{Ce Extracts Help to Maintain Central Cornea Epithelium Thickness after BAC- induced Damage}

Histological analysis showed that BAC administration generally caused cornea damage on the surface (Figure 4A), but did not reduce the total cornea thickness (Figure 4B). On the contrary, BAC caused significant increase of total cornea thickness in the peripheral cornea area (Figure 4B). The main reduction of thickness caused by BAC was on the central cornea epithelium (Figure 4C). This reduction, however, did not extend to the peripheral cornea. There was even a slight increase of epithelial thickness in the peripheral cornea (Figure 4C).
BAC administration caused increase of cornea stroma thickness in the central and peripheral cornea, but not in the limbus when compared with that of the control group (Figure 4D). With intake of Cc extracts, the thickness of stroma in the central cornea and peripheral cornea was even more increased, particularly in the central cornea where dietary intake of $\mathrm{Cc}$ extracts at $100 \mathrm{mg} / \mathrm{Kg}$ dose lead to the most significant increase. On the contrary, in the limbus, intake of Cc extracts did not increase stromal thickness (Figure 4D). At $10 \mathrm{mg} / \mathrm{Kg}$ dose, the stroma thickness in the limbus was even significantly reduced as compared to that of the BAC-damaged group.

\subsection{Moderate Mobilization of Cornea Ki-67 ${ }^{+}$ and $\mathrm{Np63}^{+}$Epithelial Cells with Cc Extracts Intake}

To further characterize the damages caused by BAC administration, we performed immunohistochemistry for $\mathrm{Ki}-67^{+}$and $\mathrm{Np} 63^{+}$cells and calculated the cell numbers in the cornea central, peripheral, and limbus regions at day 10 of the experiment. The results showed that $\mathrm{Ki}-67^{+}$cells in all of the 3 cornea regions were fewer in the blank group than in the BAC-damaged group in all of the three cornea regions (Figure 5A). Interestingly, dietary intake of Cc extracts did not appear to promote further increase of $\mathrm{Ki}-67^{+}$cells, adding to the mobilizing effect occurred after $\mathrm{BAC}$ damage. Instead, the number of $\mathrm{Ki}-67^{+}$cells became less as compared to that of the BAC-damaged group, particularly in the peripheral cornea and limbus areas when $100 \mathrm{mg} / \mathrm{Kg}$ dose was administered (Figure 5B).

A

Blank

BAC

$10 \mathrm{mg} / \mathrm{kg}$

$100 \mathrm{mg} / \mathrm{kg}$

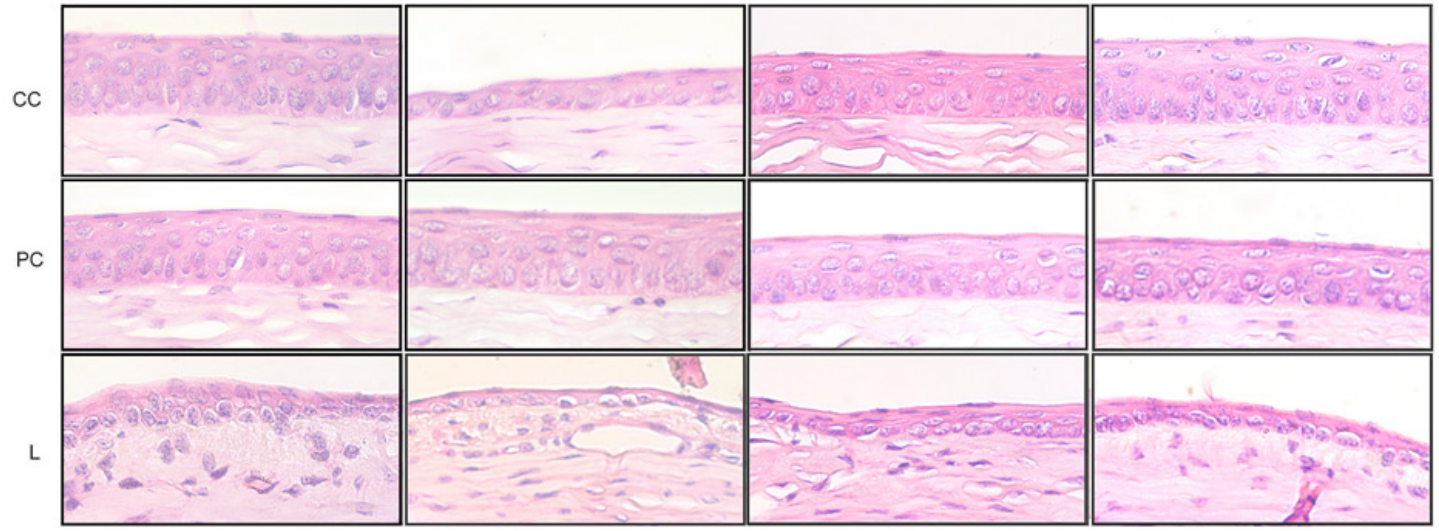

B

Total Thickness

C

Thickness of Epithelia

D

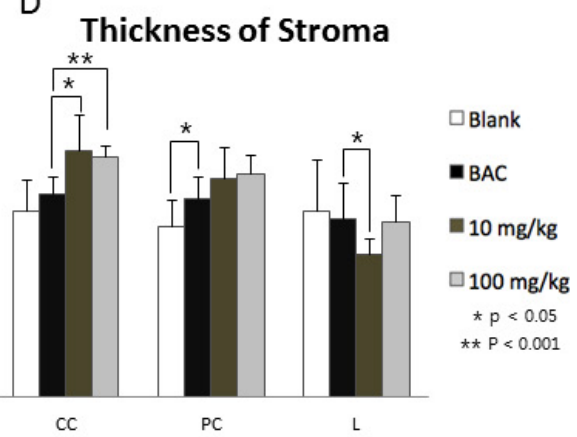

CC: central cornea. PC: peripheral cornea. L: limbus. BAC: benzalkonium chloride-damaged group without intake of Cc extracts. 10 $\mathrm{mg} / \mathrm{kg}$ and $100 \mathrm{mg} / \mathrm{kg}$ : benzalkonium chloride-damaged groups with intake of CC extracts at $10 \mathrm{mg} / \mathrm{kg}$ and $100 \mathrm{mg} / \mathrm{Kg}$, respectively.

Figure 4. Histological analysis and thickness measurement of total cornea, cornea epithelium, and cornea stroma at day 10 of the experiment after Hematoxylin-Eosin staining 


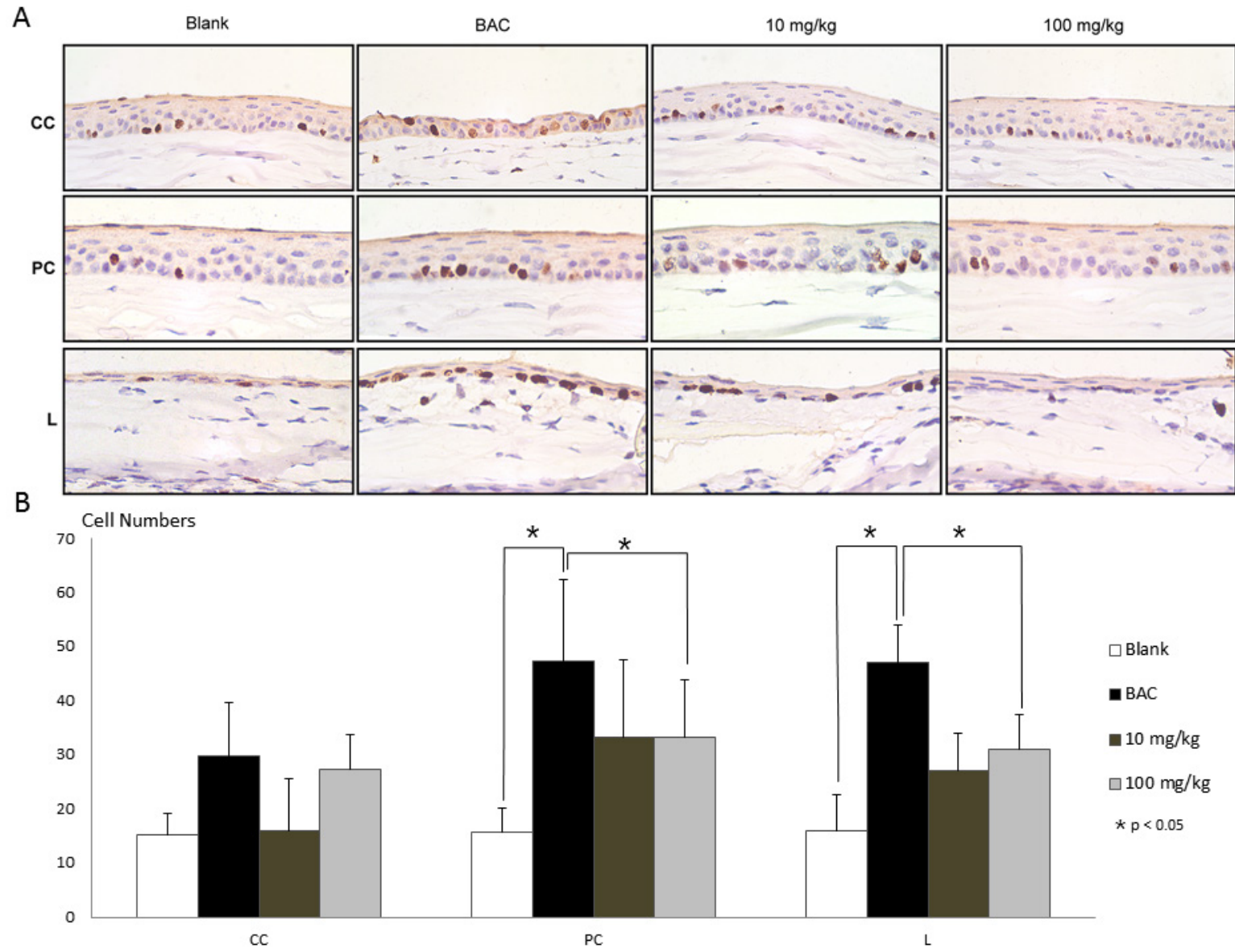

CC: central cornea. PC: peripheral cornea. L: limbus. BAC: benzalkonium chloride-damaged group without intake of CC extracts. $10 \mathrm{mg} / \mathrm{kg}$ and $100 \mathrm{mg} / \mathrm{kg}$ : benzalkonium chloride-damaged groups with intake of Cordyceps cicadae mycelia fermented extracts at $10 \mathrm{mg} / \mathrm{kg}$ and 100 $\mathrm{mg} / \mathrm{Kg}$, respectively

Figure 5. Immunohistochemical staining and quantitative analysis of Ki-67+ cells at day 10 of the experiment

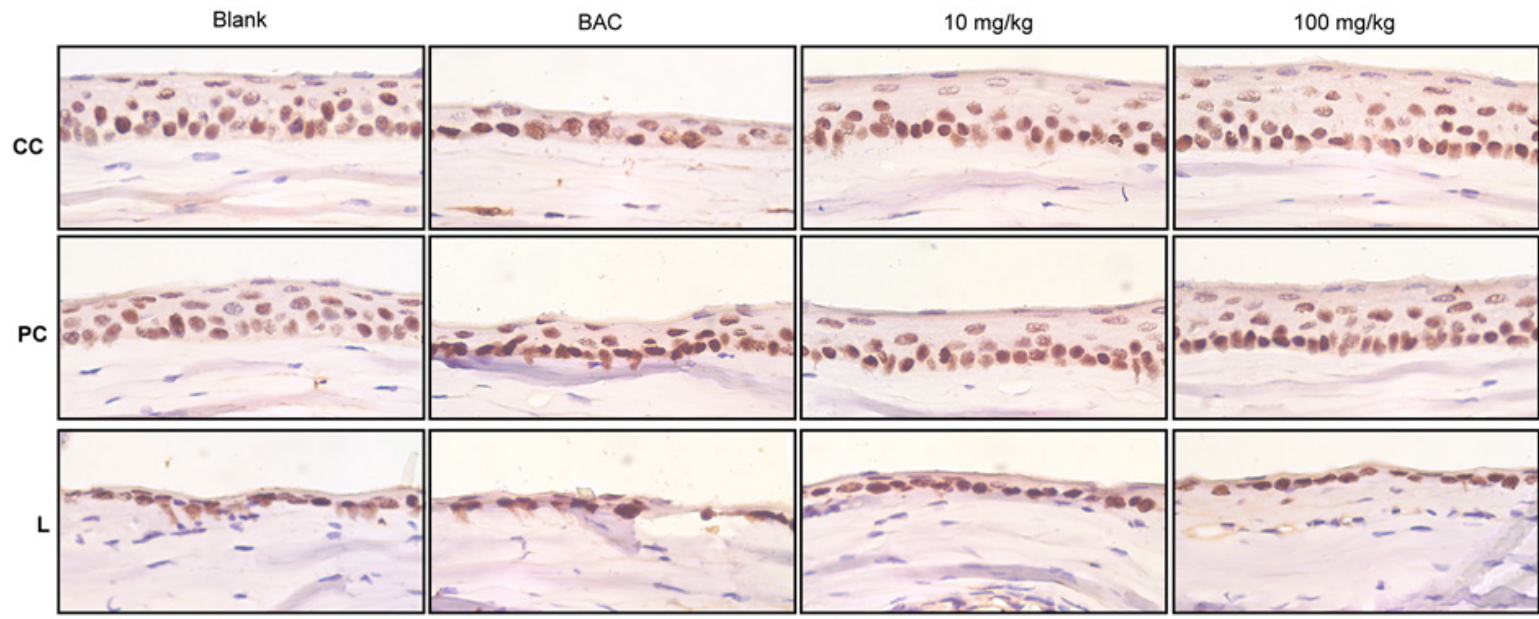

Immunohistochemical detection of $\mathrm{Np} 63^{+}$cells at day 10 of the experiment. CC: central cornea. PC: peripheral cornea. L: limbus. BAC: benzalkonium chloride-damaged group without intake of Cc extracts. $10 \mathrm{mg} / \mathrm{kg}$ and $100 \mathrm{mg} / \mathrm{kg}$ : benzalkonium chloride-damaged groups with intake of CC extracts at $10 \mathrm{mg} / \mathrm{kg}$ and $100 \mathrm{mg} / \mathrm{Kg}$, respectively

Figure 6. Immunohistochemical detection of Np63+ cells at day 10 of the experiment

In contrast to the $\mathrm{Ki}-67^{+}$cell population, the ${\mathrm{Np} 63^{+}}^{+}$ cells did not appear to be mobilized to the same extent in the peripheral cornea and limbus areas (Figure 6). When compared with that of the BAC-damaged group, the $\mathrm{Np} 3^{+}$cell number with dietary intake of Cc extracts showed no significant difference in either the $10 \mathrm{mg} / \mathrm{Kg}$ group or in the $100 \mathrm{mg} / \mathrm{Kg}$ group (data not shown).

\subsection{Cc Extracts Helps to Reduce BAC-induced Apoptosis in the Cornea Epithelium}

Since our data indicated that dietary intake of $\mathrm{Cc}$ extracts can effectively ameliorate ocular surface damages induced by BAC administration, the cornea epithelium 
must either be protected from the damages or they will die and be replaced though mobilization of limbal stem cells or basal progenitor cells [17]. Therefore, we performed TUNEL assay to examine the apoptotic status of the corneal epithelial cell populations. The results showed extensive apoptotic cells in all 3 cornea areas following BAC administration (Figure 7A). Quantitatively, the peripheral cornea area contained more apoptotic epithelial cells than the central cornea and the limbus areas following the damages induced by BAC (Figure 7B). With dietary intake of $\mathrm{Cc}$ extracts, the numbers of apoptotic cornea epithelial cells were generally reduced in both $10 \mathrm{mg} / \mathrm{Kg}$ and $100 \mathrm{mg} / \mathrm{Kg}$ study groups and the $100 \mathrm{mg} / \mathrm{Kg}$ study group apparently reduced more apoptotic epithelial cells.

\subsection{Maintenance of Conjunctival Goblet Cell with Ce Extracts Intake}

Since the tear quality was improved with intake of $\mathrm{Cc}$ extracts, as indicated by increased tear film breakup time, we performed PAS stain to assess the conjunctival goblet cell status. The results showed that $\mathrm{PAS}^{+}$ conjunctival goblet cells became fewer after the damage caused by BAC treatment (Figure 8A). With intake of $\mathrm{CC}$ extracts at $10 \mathrm{mg} / \mathrm{Kg}$, the number of $\mathrm{PAS}^{+}$ conjunctival goblet cells was higher than that in the damaged group (Figure 8B). However, less conjunctival goblet cells were observed with intake of $\mathrm{Cc}$ extracts at $100 \mathrm{mg} / \mathrm{Kg}$ dose.

\section{A}
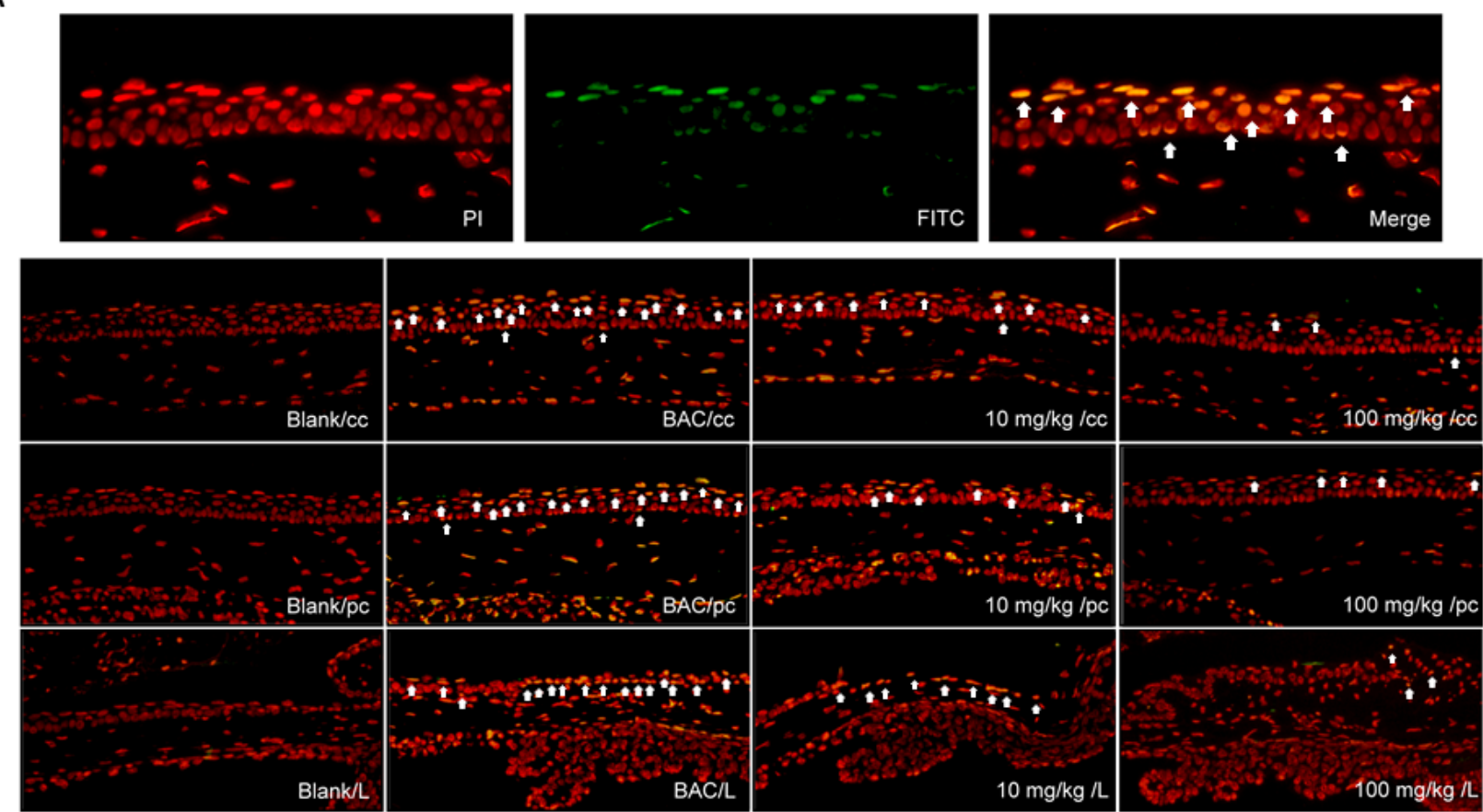

B

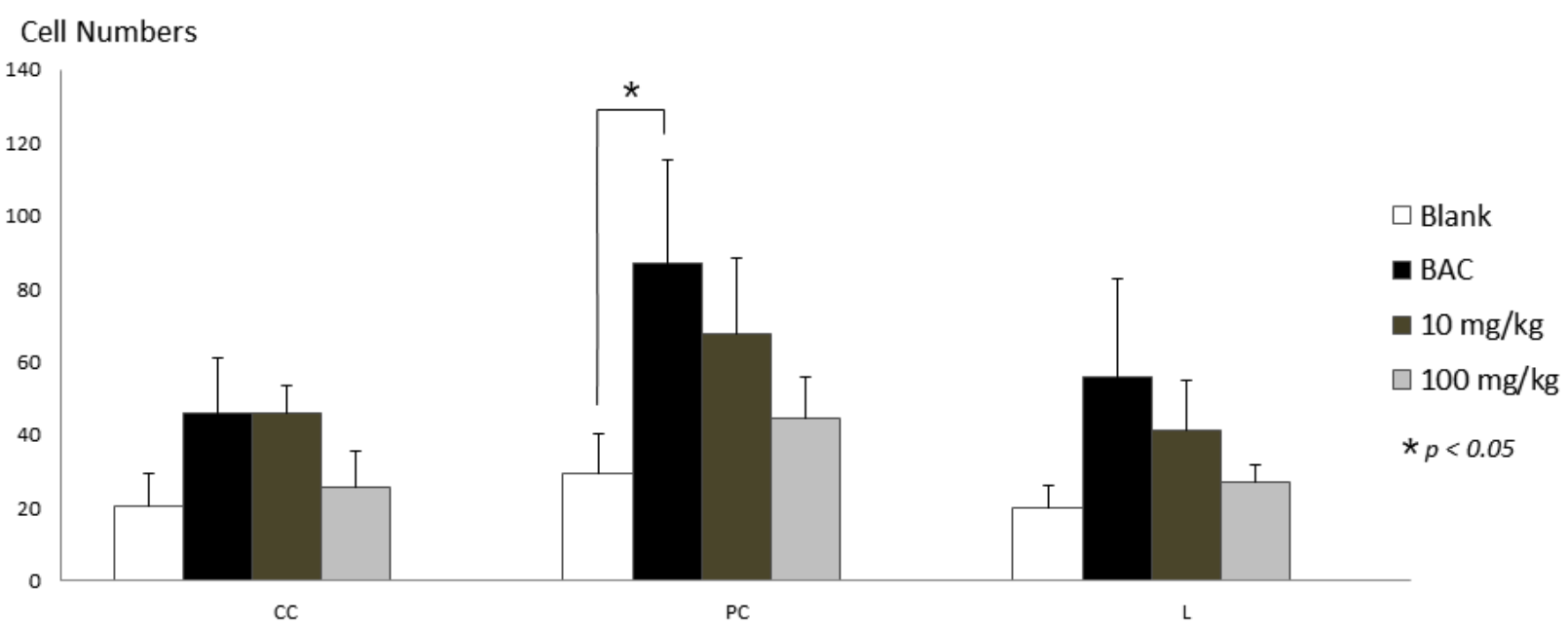

In A, the upper panel showed an example of combination of PI (in red) and FITC (in green) images to yield cells with yellow signals (indicated by arrows) that were regarded as positive for TUNEL assay. In B, quantitative analysis showed evident reduction of apoptotic cornea epithelial cells with intake of Cc extracts. CC: central cornea. PC: peripheral cornea. L: limbus. BAC: benzalkonium chloride-damaged group without intake of CC extracts. $10 \mathrm{mg} / \mathrm{kg}$ and $100 \mathrm{mg} / \mathrm{kg}$ : benzalkonium chloride-damaged groups with intake of CC extracts at $10 \mathrm{mg} / \mathrm{kg}$ and $100 \mathrm{mg} / \mathrm{Kg}$, respectively.

Figure 7. In situ terminal deoxynucleotidyl transferase dUTP nick end labeling (TUNEL) assay at day 10 of the experiment 

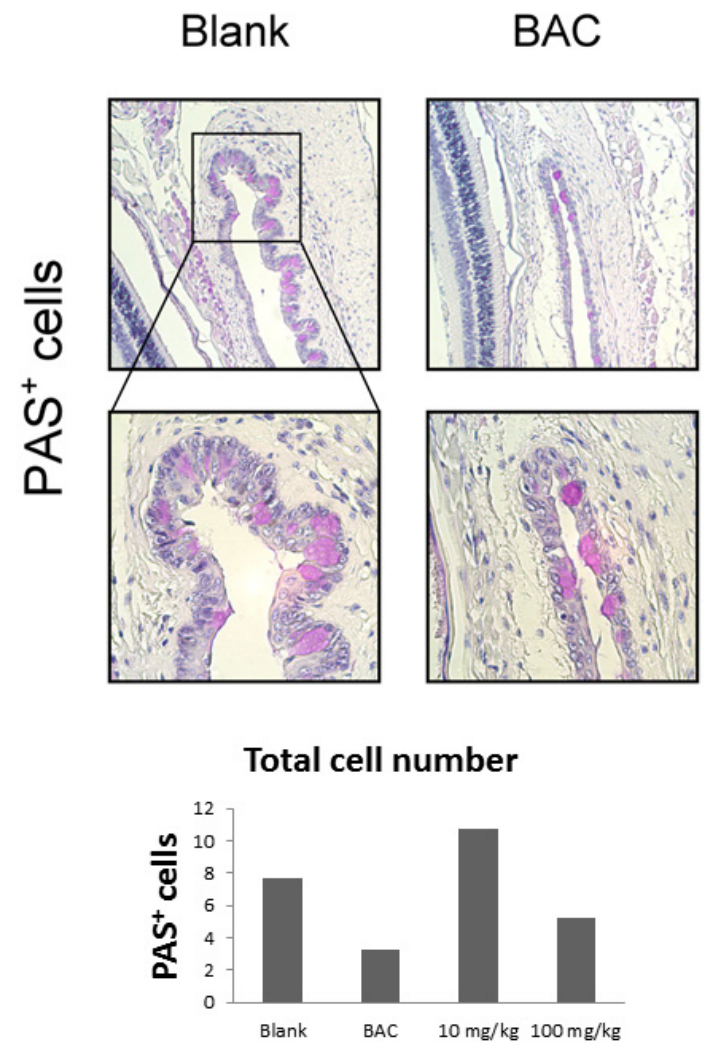

$10 \mathrm{mg} / \mathrm{kg}$
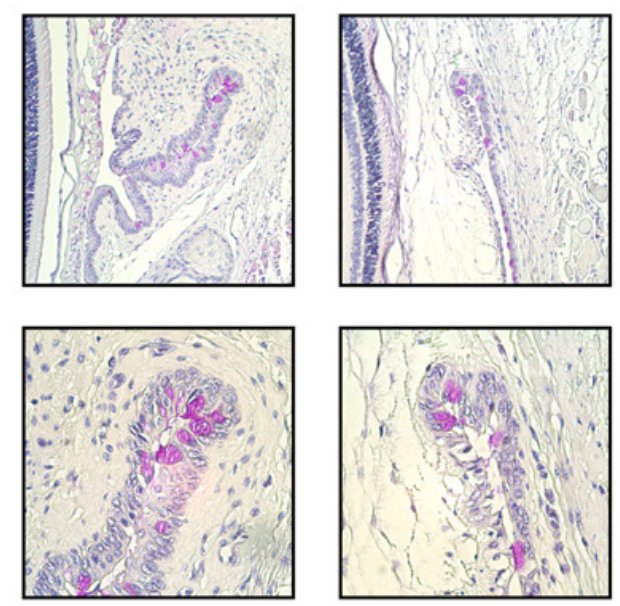

Total cell area $\left(\mu \mathrm{m}^{2}\right)$

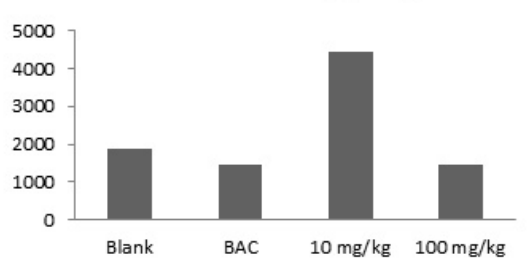

In $\mathrm{A}$, the morphology and number of goblet cells were shown in the inferior conjunctiva. In $\mathrm{B}$, the $\mathrm{PAS}^{+}$conjunctival goblet cells were quantified. The results showed that more goblet cells were maintained with intake of Cc extracts. BAC: benzalkonium chloride-damaged group without intake of CC extracts. $10 \mathrm{mg} / \mathrm{kg}$ and $100 \mathrm{mg} / \mathrm{kg}$ : benzalkonium chloride-damaged groups with intake of CC extracts at $10 \mathrm{mg} / \mathrm{kg}$ and $100 \mathrm{mg} / \mathrm{Kg}$, respectively.

Figure 8. Periodic acid Schiff (PAS) staining at day 10 of the experiment

\section{Discussion}

Dry eye disease (DED) is a multifactorial disorder of the tear film and ocular surface [3,5]. For many years, researchers have been trying to unveil the core mechanism of DED, which is reflected by the number of publications directly related to dry eye syndromes. A recent review paper [18] was published by using the key words "dry eye, inflammation" on the PubMed and Web of Science databases for scientific articles published in English between January 1, 1900 and August 30, 2013. Based on the literature survey, the authors clearly demonstrated that inflammation is the core mechanism and plays a key role in the pathogenesis of DED. The authors concluded that immune dysregulation leads to a cycle of continued inflammation and eventually leads to DED. Thus, functional foods that possess both anti-inflammatory and immunomodulatory activities may be used as candidates for alleviation of dry eye symptoms.

Cordyceps cicadae $(\mathrm{Cc})$, a caterpillar-shaped medicinal mushroom that derives its nutrients from larvae of Cicada flammata Dist., has been used as a dietary supplement in Chinese for hundreds of years. Previous studies have shown that the ergosterol peroxide from $C$. cicadae inhibits the activation and proliferation signals in primary human $\mathrm{T}$ lymphocytes [19] and ameliorates TGF- $\beta 1$-induced activation of kidney fibroblasts [14]. Another earlier study [13] indicated that C. cicadae extracts possess immunomodulatory functions. We therefore hypothesized that $C$. cicadae may help to relieve dry eye symptoms and evaluated its fermented mycelia extracts in a benzalkonium chloride (BAC)-induced mouse dry eye model. The results demonstrated that dietary intake of $\mathrm{Cc}$ extracts, particularly at $100 \mathrm{mg} / \mathrm{Kg}$ dose, helped to ameliorate BAC-induced dry eye symptoms through multiple aspects, including improvement of ocular surface grading of smoothness, topography, and lissamine green staining, increasing tear volume and TBUT period, prevention against the extensive epithelial layer apoptosis in the central, peripheral, and limbal areas of the cornea, and maintenance of conjunctival goblet cell number and area at $10 \mathrm{mg} / \mathrm{Kg}$ dose.

Multi-fold mechanisms may be unveiled underlying the efficacy after intake of $\mathrm{Cc}$ extracts. The amelioration of BAC-induced damages to the ocular surface may be through accelerated repair by mobilization of limbal stem cells or corneal progenitor cells or both $[17,20]$. Alternatively, intake of $\mathrm{Cc}$ extracts may render the corneal epithelial cells more resilient to the damages caused by BAC. We conducted immunohistochemistry of Ki-67 and Np63 cellular proliferation markers to investigate the changes of corneal epithelial cells following damages induced by $\mathrm{BAC}$ and compare the status with or without intake of Cc extracts. Ki-67 is a marker for proliferating but poorly differentiated corneal epithelial cells [21] and unspecified $\Delta \mathrm{Np} 63$ isoforms are detectable in the basal layer of the corneal epithelium at all times [21]. The results demonstrated more $\mathrm{Ki}-67^{+}$cells in all of the three cornea areas following $\mathrm{BAC}$ damage, in contrast to the steady status in the blank control group. Evidently, BAC 
damage caused more mobilization of proliferative cornea epithelial cells, either from the limbus or form the local progenitor pool.

If dietary intake of $\mathrm{Cc}$ extracts ameliorated the damages caused by BAC through accelerated cell mobilization, it would have promoted even more increase of Ki- $67^{+}$cells as compared to that of the BAC damage group without $\mathrm{Cc}$ extracts intake. Instead, $\mathrm{Ki}-67^{+}$cell number became significantly less $(\mathrm{p}<0.05$ at $100 \mathrm{mg} / \mathrm{Kg}$ dose $)$ as compared to that of the BAC-damaged group, particularly in the peripheral cornea and limbus areas. The result suggests that intake of $\mathrm{Cc}$ extracts did not help to ameliorate BAC-induced dry eye symptoms mainly through promotion for faster corneal epithelium replacement. Instead, a moderate condition of mobilization was more likely to occur. The

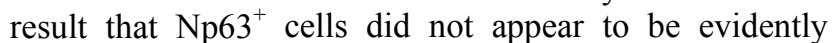
mobilized in the peripheral cornea and limbus areas also indicates this moderate condition of mobilization.

If the corneal stem cells or progenitor cells were only moderately mobilized under the effects of BAC damage, ocular surface improvement with intake of $\mathrm{Cc}$ extracts would be resulted from more resilience to the damages. The results of TUNEL assay confirmed that fewer apoptotic cells were found as Cc extracts were given, particularly in the peripheral and limbus areas when the $\mathrm{Cc}$ extracts were given at $100 \mathrm{mg} / \mathrm{Kg}$ dose.

Notably, as $\mathrm{Cc}$ extracts were given to the mice, the effective dose at $100 \mathrm{mg} / \mathrm{Kg}$ correlates in almost all aspects of dry eye symptom amelioration, except for the cell number and area of conjunctival goblet cells. Significant amelioration was observed at $100 \mathrm{mg} / \mathrm{Kg}$ dose for 3 out of the 4 ocular surface grading criteria. The results of tear production and TBUT were also better with $100 \mathrm{mg} / \mathrm{Kg}$ dose. Another result that is also noteworthy is the significant increase of stromal thickness in the central cornea, in contrast to the non-significance in the peripheral cornea and the limbus. This data is conversely correlated with the non-significant change of $\mathrm{Ki}-67^{+}$cell number in the central cornea and the significant change in the peripheral cornea and in the limbus. Interestingly, fewer apoptotic cornea epithelial cells were detected in the central cornea in the BAC-damaged group as well as in the groups given $\mathrm{Cc}$ extracts. The reduction of central cornea epithelial apoptotic activity is likely due to the more increase of stromal thickness in the central cornea where differential corneal epithelial-keratocyte cell interactions may occur in this area, leading to differential release of protective factors from the keratocytes. For example it has been known that paracrine mediators such as hepatocyte growth factor (HGF) and keratinocyte growth factor (KGF) are produced by the keratocytes to regulate proliferation, motility, differentiation, and possibly other functions, of epithelial cells $[22,23]$. If the differential epithelial-keratocyte cell interaction occurred, since the epithelial cells in the central cornea were more protected, apoptotic activities were relatively low and the loss of central cornea epithelial cells would be less. The demand for repair would be less, as reflected by the non-significant change of $\mathrm{Ki}-67^{+}$cell number in the central cornea. Arguably, however, increase of the central stroma thickness may be an indication of edema caused by inflammatory activities therein. Besides, the effects to maintain conjunctival goblet cell were better with $\mathrm{Cc}$ extracts at $10 \mathrm{mg} / \mathrm{Kg}$ than at $100 \mathrm{mg} / \mathrm{Kg}$ dose. Complicated local factors may be involved, resulting in impedance of goblet cell generation with Cc extracts given at higher dose. Obviously, this entire scenario demands further investigations.

The underlying mechanisms between maintenance of healthy cornea epithelial layers and improvement of tear production and quality are multifold. Particularly, since $\mathrm{Cc}$ extracts possess both immunomodulatory and anti-inflammatory activities, the ameliorating effects found in the present study are of complexity and warrant further investigation. Also, the clinical significance between protecting corneal epithelium from extensive apoptosis rather than acceleration of stem cell mobilization and replacement of damaged cells is different. To the aged corneas or corneas with limbal stem cell deficiency, as their cornea stemness capacity is diminished, stimulation to accelerate cornea repair is less likely to ameliorate dry eye symptoms. Under these conditions, dietary Cc extracts are potentially more helpful.

\section{Materials and methods}

\subsection{Mice and Study Groups}

A total of 24 six-week-old female ICR mice were purchased from BioLASCO Taiwan Co., Ltd, Taipei, Taiwan. The mice were fed ad libitum and kept under standard conditions with a 12-h light/dark cycle. The mice were acclimatized and habituated to the laboratory for at least one week before experiments. All mice were examined with a slit lamp (Model 99 BQ; Haag-Streit, Bern, Switzerland). Only mice without anomalies of the anterior segment of the eye (cornea, anterior chamber, iris, or lens) were included in the experiments. The mice were randomly split into 4 groups: blank with $0.9 \% \mathrm{NaCl}$ as vehicle control, BAC-damaged without $\mathrm{CC}$ extracts, BAC-damaged with $10 \mathrm{mg} / \mathrm{kg}$ bodyweight of Cc extracts, BAC-damaged with $100 \mathrm{mg} / \mathrm{kg}$ bodyweight of Cc extracts. Each group contained 6 mice. All experiment protocols were reviewed and approved by the Animal Care and Use Committee of Chung Shan Medical University, Taichung, Taiwan and were performed in agreement with the Association for Research in Vision and Ophthalmology (ARVO) Resolution on the Use of Animals in Research.

\subsection{Cornea Surface Damage by Benzalkonium Chloride Administration}

The BAC damages were performed from day 1 to day 10 , with the mice anaesthetized and their ocular surfaces exposed to $5 \mu \mathrm{L}$ of $0.2 \%$ BAC administered topically twice daily (9 AM and 9 PM). Care was taken to ensure that BAC covered the entire mouse ocular surface for at least 1 minute during each BAC administration.

\subsection{Preparation and Feeding of Cc Extracts}

Cordyceps cicadae was harvested from mountain areas of New Taipei City, Taiwan. The mycelium was isolated and cultured on potato dextrose agar and genotyped to 
confirmed its identity. The colony has been stored (stock number MU30106) in Bioresource Collection and Research Centre (BCRC), Hsinchu, Taiwan. The mycelium was primarily cultured at $25^{\circ} \mathrm{C}$ in $2 \%$ glucose, $1 \%$ yeast extract, $1 \%$ soy bean powder at $\mathrm{pH} 6.0$, followed by expanded culture and fermentation. The crude fermented fluids were concentrated, cold-dried into powder, and stored at $4^{\circ} \mathrm{C}$ before feeding. The $\mathrm{Cc}$ extracts were given by daily tube feeding at 10 AM starting from 3 days prior to the BAC damage and continuing until day 10 . All mice were sacrificed on day 10 for tissue collection.

\subsection{Grading of Corneal Surface Damages}

Corneal surface damages were graded according to smoothness, topography, opacity, and the extent of lissamine green staining, following a previous publication [10]. Briefly, one eye of each mouse was randomly selected for assessment of corneal smoothness and topography. The other eye was then assessed for corneal opacity. Images of the cornea surface were taken with a stereoscopic zoom microscope equipped with ring illuminator (SMZ 1500; Nikon). The digital images were then used to score corneal smoothness on a 5-point scale according to the number of distorted quadrants in the reflected ring: 0, no distortion; 1, distortion in one quadrant of the ring ( 3 clock hours); 2 , distortion in two quadrants (6 clock hours); 3 , distortion in three quadrants (9 clock hours); 4, distortion in all four quadrants (12 clock hours); and 5, severe distortion, in which no ring could be recognized. The scoring of corneal topography was the same as that of corneal smoothness, but covered much more area of the corneal surface. The other set of images on the other eye was used for corneal opacity scoring $(0$, normal cornea; 0.5 , mild haze seen only under dissection microscope; 1, mild haze; 2, moderate haze with visible iris; 3, severe haze with invisible iris; 4, severe haze with corneal ulceration). For lissamine green staining, both corneas from each mouse were stained with 2\% lissamine green B (Sigma-Aldrich, cat. no. 199583) in $0.9 \% \mathrm{NaCl}$. Images were taken and scored according to the areas of stain. Briefly, the total area of punctuate staining was designated as grade 0 ; grade 1, less than $25 \%$ of cornea stained with scattered punctuate staining; grade $2,25 \%$ to $50 \%$ of cornea stained with diffuse punctate staining; grade $3,50 \%$ to $75 \%$ of cornea stained with punctuate staining and apparent epithelial defects; grade 4, more than $75 \%$ of cornea stained with abundant punctuate staining and large epithelial defects. All scorings were performed by two observers without prior knowledge of the experiment and study groups.

\subsection{Tear Production and Tear Film Breakup Time Assessment}

Aqueous tear production was assessed with phenol red-impregnated cotton threads (Zone-Quick; Oasis, Glendora, CA, USA) at days 1, 4, 7, and 10. The animals were anesthetized and rest for a fixed period of 20 seconds. A 1-mm-width strip was held with forceps under a dissection microscope and placed in the lateral cantus of the conjunctival fornix of the eye for 20 seconds. The tear distance (in millimeters) was read under a microscope.
Tear film breakup time was assessed by in vivo staining with $0.1 \%$ liquid sodium fluorescein $[10,16]$. The mice were anesthetized with intraperitoneal tribromoethanol injection $(0.3 \mathrm{ml}$ at $20 \mathrm{mg} / \mathrm{ml})$. One or two microliter of fluorescein stain was dropped into the conjunctival sac. After 3 blinks, tear film fluorescein signals were recorded under an Olympus BX51 fluorescence microscope (Olympus, Tokyo, Japan). The tear film breakup time was read in seconds by two observers without prior knowledge of the experiment and study groups.

\subsection{Histology and Immunohistochemistry Analysis}

After mouse sacrifice by cervical dislocation, the eyes were extracted, processed, and stained with Hematoxylin-Eosin for histopathologic analysis following conventional procedures as previously described $[10,16]$. For immunohistochemistry, the tissue sections were boiled in citrate buffer ( $\mathrm{pH}$ 6.0) for 20 minutes for antigen retrieval and then incubated, respectively, with either mouse anti-Np63 (1/50, cat. no. sc-8431; Santa Cruz Biotechnology, Santa Cruz, CA) or rabbit anti-Ki-67 antibody (1/100, cat. no. NB110-89719; Novus Biologicals), followed by incubation with a horseradish peroxidaseconjugated secondary antibody (1/200), either anti-mouse or anti-rabbit IgG (Jackson ImmunoResearch Laboratories, Inc., West Grove, PA), and followed by washes and incubation in diaminobenzidine tetrahydrochloride solution for color detection, and counterstained with hematoxylin.

\subsection{Demarcation of Central, Peripheral, and Limbal Cornea Areas and Thickness Measurement}

To describe differential cell distribution and corneal thickness in different areas, each cornea was demarcated into central, peripheral, and limbal areas. The eye was sectioned on sagittal plane and the cornea length, spanning from the upper to the lower limbus, was split into 3 equal parts. The upper one third and the lower one third were regarded as the peripheral corneas, while the middle one third as the central cornea. Following Hematoxylin-Eosin stain, the tissue sections with the longest corneal length were selected and measured for total, epithelial, and stromal thickness under a Nikon E 100 microscope (Nikon, Tokyo, Japan), equipped with a digital camera linked to a desktop computer. Each thickness measurement was determined by 2 observers without prior information of the experiments and study groups. All measurements were performed on ImageJ2 (National Institutes of Health, Bethesda, USA) software program.

\subsection{In Situ Terminal Deoxynucleotidyl Transferase dUTP Nick End Labeling (TUNEL) Assay}

In situ TUNEL labeling was performed using the DeadEnd ${ }^{\mathrm{TM}}$ Fluorometric TUNEL System (G3250; Promega, Madison, WI) according to manufacturer's instructions. Tissue sections were fixed in acetone at $4{ }^{\circ} \mathrm{C}$, rinsed with phosphate-buffer saline (PBS), permeabilized 
by $0.2 \%$ Triton $\mathrm{X} 100$, and incubated in equilibration buffer for $10 \mathrm{~min}$. The sections were then incubated with TdT reaction mix for $60 \mathrm{~min}$, followed by immersion in standard saline citrate to stop reaction. For positive controls, tissue sections were incubated in DNase I prior to addition of equilibration buffer. For negative controls, DDW was added instead of TdT reaction mix. All preparations were rinsed with PBS several times, counterstained with propidium iodide to locate the cells (P3566; ThermoFisher), and mounted for photography. The photo images were taken with an Olympus BX51 fluorescence microscope (Olympus, Tokyo, Japan), followed by digital combination of the fluorescein isothiocyanate (FITC) (green) with the propidium iodide (red) color to produce yellow signals. Only cells with yellow signals were regarded as positive for TUNEL assay.

\subsection{PAS Stain for Conjunctival Goblet Cell Number and Total Cell Areas}

Periodic acid Schiff (PAS) staining was performed to evaluate conjunctival epithelial morphology, and the number of goblet cells and total cell areas in the inferior conjunctiva was counted under a microscope (ECLIPSE E100; Nikon, Melville, NY) with a $\times 20$ objective and ImageJ2 software program. For each eye, 5 different sections were randomly selected for counting, and an average was calculated. All scorings were performed by two observers without prior knowledge of the study groups.

\subsection{Statistics}

All data were obtained from repeats $(n \geq 6)$. The data are presented as the means \pm standard error of the means (SEMs) and were compared among groups. The corneal smoothness, opacity, and fluorescein staining scores were compared with the Kruskal-Wallis test. The corneal total, epithelial, and stromal thickness and the number of cells positive in immunohistochemistry and TUNEL assay were analyzed with the Mann-Whitney test. All statistical analyses were performed by using the SPSS program (SPSS, Inc., Chicago, IL).

\section{Conclusion}

We demonstrated that $C$. cicadae fermented mycelia extracts help to ameliorate dry eye symptoms after ocular surface damages caused by benzalkonium chloride administration. The efficacy was shown in multiple aspects, including tear quality and quantity as well as ocular surface assessments. Furthermore, we elucidated that $C$. cicadae fermented mycelia extracts help to ameliorate the dry eye symptoms through enhancement of cornea resilience and maintenance of conjunctival goblet cells after benzalkonium chloride administration. Results of this study support that C. cicadae fermented mycelia extracts may be used as a functional food ingredient for vision protection.

\section{Conflicts of Interests}

The authors declare no conflict of interest.

\section{Author Contributions}

D.P.-C.L. and H.-H.C conceived and designed experiments and wrote the paper. T.-Y.L. and Y.-J.T. performed experiments. C. -C.C. and L.-Y.L. provided materials and reagents and analyzed the data.

\section{Acknowledgements}

This study was supported by research grants: MOST 102-2320-B-040-013 from Ministry of Science and Technology, Taiwan; COA 104-20-26 and COA 105A005-C from Council of Agriculture, Taiwan.

\section{References}

[1] Lin, P. Y.; Tsai, S. Y.; Cheng, C. Y.; Liu, J. H.; Chou, P.; Hsu, W. M. Prevalence of dry eye among an elderly Chinese population in Taiwan: the Shihpai Eye Study. Ophthalmology. 2003; Volume 110, pp. 1096-1101.

[2] O’Brien, P. D.; Collum, L. M. Dry eye: diagnosis and current treatment strategies. Curr. Allergy Asthma. Rep. 2004; Volume 4, pp. 314-319.

[3] Dry Eye WorkShop. Research in dry eye: report of the Research Subcommittee of the International Dry Eye Workshop. Ocul. Surf. 2007; Volume 5, pp. 179-193.

[4] Arevalo, J. F.; Lowder, C. Y.; Muci-Mendoza, R. Ocular manifestations of systemic lupus erythematosus. Curr. Opin. Ophthalmol. 2002; Volume 13, pp. 404-410.

[5] Yen, J. C.; Hsu, C. A.; Li, Y. C.; Hsu, M. H. The Prevalence of dry eye syndrome's and the likelihood to develop Sjögren's syndrome in Taiwan: A Population-Based Study. Int. J. Environ. Res. Public Health. 2015; Volume 12, pp. 7647-7655.

[6] Cordero-Coma, M.; Anzaar, F.; Sobrin, L.; Foster, C. S. Systemic immunomodulatory therapy in severe dry eye secondary to inflammation. Ocul. Immunol. Inflamm. 2007; Volume 15, pp. 99-104.

[7] Huang, J. F.; Yafawi, R.; Zhang, M.; McDowell, M.; Rittenhouse, K. D.; Sace, F.; Liew, S. H.; Cooper, S. R.; Pickering, E. H. Immunomodulatory effect of the topical ophthalmic Janus kinase inhibitor tofacitinib (CP-690,550) in patients with dry eye disease. Ophthalmology. 2012; Volume 119, pp. e43-50.

[8] Zheng, Q.; Ren, Y.; Reinach, P. S.; Xiao, B.; Lu, H.; Zhu, Y.; Qu, J.; Chen, W. Reactive oxygen species activated NLRP3 inflammasomes initiate inflammation in hyperosmolarity stressed human corneal epithelial cells and environment-induced dry eye patients. Exp. Eye Res. 2015; Volume 34, pp. 133-140.

[9] Deng, R.; Hua, X.; Li, J.; Chi, W.; Zhang, Z.; Lu, F.; Zhang, L.; Pflugfelder, S. C.; Li, D. Q. Oxidative stress markers induced by hyperosmolarity in primary human corneal epithelial cells. PLoS One. 2015; pp. e0126561.

[10] Chen, B. Y.; Lin, D. P.; Chang, L. S.; Huang, T. P.; Liu, H. J.; Luk, C. P.; Lo, Y. L.; Chang, H. H. Dietary $\alpha$-lipoic acid prevents UVB-induced corneal and conjunctival degeneration through multiple effects. Invest. Ophthalmol. Vis. Sci. 2013; Volume 54, pp. 6757-6766.

[11] Andrade, A. S.; Salomon, T. B.; Behling, C. S.; Mahl, C.D.; Hackenhaar, F. S.; Putti, J.; Benfato, M. S. Alpha-lipoic acid restores tear production in an animal model of dry eye. Exp. Eye Res.2014; Volume 120, pp. 1-9.

[12] Mencucci, R.; Favuzza, E.; Boccalini, C.; Lapucci, A.; Felici, R.; Resta, F.; Chiarugi, A.; Cavone, L. CoQ10-containing eye drops prevent UVB-induced cornea cell damage and increase cornea wound healing by preserving mitochondrial function. Invest. Ophthalmol. Vis. Sci. 2014; Volume 55, pp. 7266-7271.

[13] Weng, S. C.; Chou, C. J.; Lin, L. C; Tsai, W. J.; Kuo, Y. C. Immunomodulatory functions of extracts from the Chinese medicinal fungus Cordyceps cicadae. J. Ethnopharmacol. 2002; Volume 83, pp. 79-85.

[14] Zhu, R.; Zheng, R.; Deng, Y.; Chen, Y.; Zhang, S. Ergosterol peroxide from Cordyceps cicadae ameliorates TGF- $\beta 1$-induced 
activation of kidney fibroblasts. Phytomedicine. 2014; Volume 21, pp. 372-378.

[15] Lin, Z.; Liu, X.; Zhou, T.; Wang, Y.; Bai, L.; He, H.; Liu Z. A mouse dry eye model induced by topical administration of benzalkonium chloride. Mol. Vis. 2011; Volume 17, pp. 257-264.

[16] Lin, D. P.; Chang, H. H.; Yang, L. C.; Huang, T. P.; Liu, H. J.; Chang, L. S.; Lin, C. H.; Chen, B. Y. Assessment of ultraviolet B-blocking effects of weekly disposable contact lenses on corneal surface in a mouse model. Mol. Vis. 2013; Volume 19, pp. 1158-1168.

[17] Majo, F.; Rochat, A.; Nicolas, M.; Abou Jaoudé, G.; Barrandon, Y. Oligopotent stem cells are distributed throughout the mammalian ocular surface. Nature. 2008; Volume 456, pp. 250-254.

[18] Wei, Y.; Asbell, P. A. The core mechanism of dry eye disease (DED) is inflammation. Eye Contact Lens. 2014; Volume 40, pp. 248-256.

[19] Kuo, Y. C.; Weng, S. C.; Chou, C. J.; Chang, T. T.; Tsai, W. J. Activation and proliferation signals in primary human $\mathrm{T}$ lymphocytes inhibited by ergosterol peroxide isolated from Cordyceps cicadae. Br. J. Pharmacol. 2003; Volume 140, pp. 895-906.

[20] Yoon, J. J.; Ismail, S.; Sherwin, T. Limbal stem cells: central concepts of corneal epithelial homeostasis. World J. Stem Cells. 2014; Volume 6, pp. 391-403.

[21] Pearton, D. J.; Yang, Y.; Dhouailly, D. Transdifferentiation of corneal epithelium into epidermis occurs by means of a multistep process triggered by dermal developmental signals. Proc. Natl. Acad. Sci. USA. 2005; Volume 102, pp. 3714-3719.

[22] Wilson, S. E.; Liu, J. J.; Mohan, R. R. Stromal-epithelial interactions in the cornea. Prog. Retin. Eye Res. 1999; Volume 18, pp. 293-309.

[23] Ramaesh, T.; Ramaesh, K.; Martin Collinson, J.; Chanas, S. A.; Dhillon, B.; West, J. D. Developmental and cellular factors underlying corneal epithelial dysgenesis in the Pax6+/- mouse model of aniridia. Exp. Eye Res. 2005; Volume 81, pp. 224-35. 